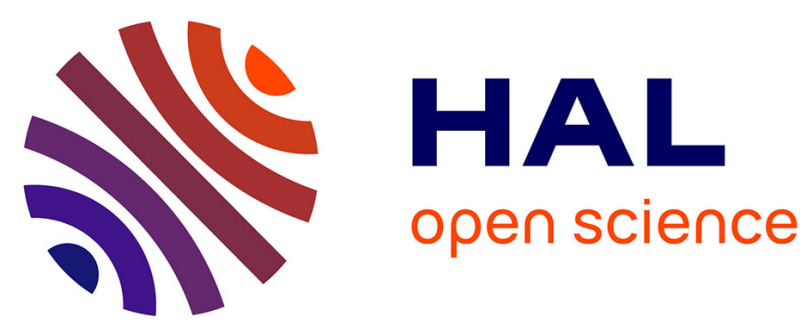

\title{
Ergonomic and Gesture Performance of Robotized Instruments for Laparoscopic Surgery
}

Benoît Herman, Ali Hassan Zahraee, Jérome Szewczyk, Guillaume G. Morel, Christophe Bourdin, Jean-Louis Vercher, Brice Gayet

\section{- To cite this version:}

Benoît Herman, Ali Hassan Zahraee, Jérome Szewczyk, Guillaume G. Morel, Christophe Bourdin, et al.. Ergonomic and Gesture Performance of Robotized Instruments for Laparoscopic Surgery. 2011 IEEE/RSJ INTERNATIONAL CONFERENCE ON INTELLIGENT ROBOTS AND SYSTEMS, 2011, 345 E 47TH ST, NEW YORK, NY 10017 USA, United States. pp.1333-1338. hal-01436023

\section{HAL Id: hal-01436023 \\ https://hal.science/hal-01436023}

Submitted on 26 Apr 2018

HAL is a multi-disciplinary open access archive for the deposit and dissemination of scientific research documents, whether they are published or not. The documents may come from teaching and research institutions in France or abroad, or from public or private research centers.
L'archive ouverte pluridisciplinaire HAL, est destinée au dépôt et à la diffusion de documents scientifiques de niveau recherche, publiés ou non, émanant des établissements d'enseignement et de recherche français ou étrangers, des laboratoires publics ou privés. 


\title{
Ergonomic and Gesture Performance of Robotized Instruments for Laparoscopic Surgery
}

\author{
Benoît Herman, Ali Hassan Zahraee, Jérôme Szewczyk, Guillaume Morel, \\ Christophe Bourdin, Jean-Louis Vercher and Brice Gayet
}

\begin{abstract}
Shape and mechanical structure of instruments play a large part in the lack of ergonomics during laparoscopic surgery. Providing intra-abdominal mobility and rethinking handles design are two solutions to increase comfort and precision of gestures. Based on previous work that determined the optimal intra-abdominal kinematics, this study analyses the influence of handle design on both gesture and ergonomic performance. A virtual reality laparoscopic simulator was developed to perform an experimental comparison between two novel robotized instruments and standard ones. A group of 10 surgeons and 6 researchers in robotics carried out two representative surgical tasks with each instrument. Based on instrument and arm segments tracking, a gesture performance index and an ergonomic performance index were computed. The study demonstrates that distal mobilities combined with improved handle design and integration increase ergonomic level during laparoscopy and facilitate complex gestures.
\end{abstract}

\section{INTRODUCTION}

Many studies in the operating room and on pelvitrainers or simulators, along with large surveys of surgeons, demonstrated that laparoscopists work in poorly ergonomic conditions. Several reasons are pointed out: 2D monoscopic vision on screen, indirect vision of hands and unnatural line of sight, inversion and variable scaling of motions, reduced intra-abdominal mobility due to the fulcrum effect of the small incisions [1]-[3].

The elongated shape and mechanical structure of standard laparoscopic instruments are also implicated in those problems [4], [5]. The orientation of the handle (pistol-shaped) with respect to the shaft and the length of the latter increase the movement amplitude of the upper limb and the strain in the surgeon's shoulders, elbows and, above all, wrists. The unusual posture of upper limbs combined with the weak mechanical efficiency (friction) in the opening mechanism of instruments are reported to increase muscles recruitment.

This work was supported by Agence Nationale de la Recherche grant ANR-09-CORD-020.

B. Herman, A.H. Zahraee, J. Szewczyk and G. Morel are with UPMC Univ Paris 06, UMR 7222, ISIR, F-75005, Paris, France and CNRS, UMR 7222, ISIR, F-75005, Paris, France \{zahraee, szewczyk, morel\}@isir.upme.fr

B. Herman is now F.R.S.-FNRS Postdoctoral Researcher with Universite catholique de Louvain, Center for Research in Mechatronics, B-1348 Louvain-la-Neuve, Belgium benoit.herman@uclouvain.be

C. Bourdin and J.L. Vercher are with Université de la Méditerranée, UMR 6233, ISM, F-13288, Marseille, France and with CNRS, UMR 6233, ISM, F-13288, Marseille, France \{christophe.bourdin, jean-louis.vercher $\}$ aunivmed. fr

B. Gayet is with Department of Medical and Surgical Digestive Disease, Institut Mutualiste Montsouris, Université Paris Descartes, F-75014, Paris, France brice.gayeteimm.fr
This overall lack of ergonomics in laparoscopy is at the source of pain and feeling of discomfort [2], [3], [6]. Most of these aches are due to the increase of muscular efforts in the upper limb. Wrist and elbow tendinitis are rather common among experienced laparoscopists because of the awkward arm postures they are used to take-elbows raised and pointing outwards or even upwards, wrists bent and twisted excessively. Furthermore, neck and back stiffness and fatigue often occurs, due to the static position of the trunk and head of the surgeon who looks at the screen most of the time. Finally, most instruments are closed by thumb and the application of a high force on a small contact surface compresses one of the medial nerve endings. This often causes thumb numbness that may spread to the whole hand.

Various tracks have been followed for the last decade to improve comfort and raise the quality of laparoscopic surgery. In addition to the broad diffusion of instructions (e.g. trocars placement, table adjustment, spatial organization of monitors and surgical team) for operating in the most adequate posture, an increasing interest is taken in the development of novel instruments. In particular, industrials and researchers work on two main topics: providing additional intra-abdominal mobility by adding several joints at the distal tip of instruments, and designing more ergonomic handles [7]. A comprehensive review of manual and robotized articulated instruments can be found in [8].

Intra-abdominal degrees of freedom (DOF) mainly intend to increase quality and precision of several gestures (e.g. blood vessels cut and coagulation, clip placement, intracorporeal stitch and knot) that are rather difficult to perform with the only 4 DOF of conventional instruments. In [9], we compared several 2 and 3 DOF distal kinematics through suturing trials on a virtual reality (VR) simulator that was developed for the purpose. The most appropriate kinematics (i.e. Yaw-Roll) was then implemented in several prototypes.

As a supplement to this initial study, the present paper focuses on the design of an ergonomic handle and its integration to the articulated instrument, so as to allow the surgeon to work in a more comfortable posture and to control intuitively the additional mobilities.

\section{Materials And Methods}

To assess the influence of distal DOFs and handle design on gesture and ergonomic performance in laparoscopic surgery, an experimental protocol was set up. Using a VR simulator, novel instruments were compared with standard graspers through two representative tasks. 

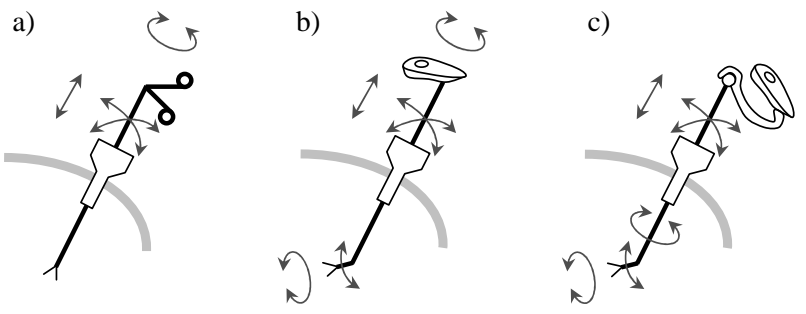

Fig. 1. Kinematics of the three instruments that were compared experimentally: a) standard instrument; b) robotized instrument with 2 intra-abdominal DOF and fixed handle; c) robotized instrument with 2 intra-abdominal DOF, active trocar and moving handle

\section{A. Instruments}

The 3 instruments depicted on Fig. 1 were evaluated in this study. In addition to two robotized instruments each featured with a controllable Yaw-Roll intra-abdominal part (described below), the subjects also performed the exercises with standard instruments, in order to quantify the advantage of the new instruments over current ones.

To give the surgeon a direct and intuitive control of the additional tip mobilities, a Wii Nunchuk is used as handle. It is designed ergonomically, so the user has a good grip on it. Its 2-DOF joystick can be used to control the actuated intraabdominal motions. The Nunchuk handle can be fixed on the shaft (see Fig.1b) with an angle that maximizes ergonomic performance and gesture quality, according to [10].

However, the intra-abdominal workspace is large. As a consequence, ergonomic level of the instantaneous posture highly depends on the intra-abdominal region where the surgeon works. Indeed, some orientations of the instrument shaft impose an awkward arm configuration with often high flexion and deviation of the wrist. To solve this problem, we introduced in [8] an alternative version of the robotized instrument that comprises a free spherical joint between handle and shaft (see Fig.1c). This passive joint releases the kinematic constraints on the wrist and allows to cover the whole intra-abdominal workspace while keeping the hand in line with the forearm.

The presence of this 3-DOF joint implies that the handle can turn freely around the instrument longitudinal axis without turning the shaft. This motion has therefore to be motorized. As detailed in [8], a motor is embedded in a part of the instrument called active trocar that is tightened on a standard trocar. It allows a free manual translation of the instrument inside the trocar but controls the rotation of the shaft that cannot be performed manually. Two buttons of the Nunchuk (additionals to the joystick) are used to turn the instrument around its axis.

\section{B. Surgical Tasks}

In order to quantify the performance of laparoscopists, many studies have suggested over the last decade various protocols for evaluation of laparoscopic skills during and after training. These exercises can be performed on a pelvitrainer, a VR simulator or in vivo using animal models. Derossis et al. [11] proposed a series of 7 exercises that cover the whole range of gestures performed by surgeons during any laparoscopy: grasping an object and passing it from one instrument to the other, dissecting and cutting along a prescribed trajectory, clipping and cutting a vessel, ligaturing a vessel with a thread, placing and stitching a mesh, suturing with an intra-corporeal knot, and suturing with an extracorporeal knot. Cao et al. [12] analyzed laparoscopic gestures from a kinematic point of view and identified 5 motions or actions: pointing and reaching a target and orienting the effector, closing the effector to grasp, hold or cut, pushing with the instrument, pulling, and opening the effector.

For practical reasons, we tried to minimize the number of tasks to perform during the experiments. We selected 2 exercises from Derossis' set: a pointing task and a pick and place task. They cover most of Cao's motions and actions, and are pretty representative of laparoscopic gestures. Furthermore, they are not too long and can be repeated several times.

Pointing task (see Fig. 2a): it consists in moving the instrument tip in free path and touching targets (blue sphere in Fig. 2a) that appear successively in the workspace. This task does not require the intra-abdominal DOFs (that are therefore disabled) and essentially focus on the handle influence on ergonomic performance and motion accuracy.

Pick and place task (see Fig. 2b-c): the user has to pick a ring and place it on a pin in another position. This is a more complex task than pointing that requires hand and wrist
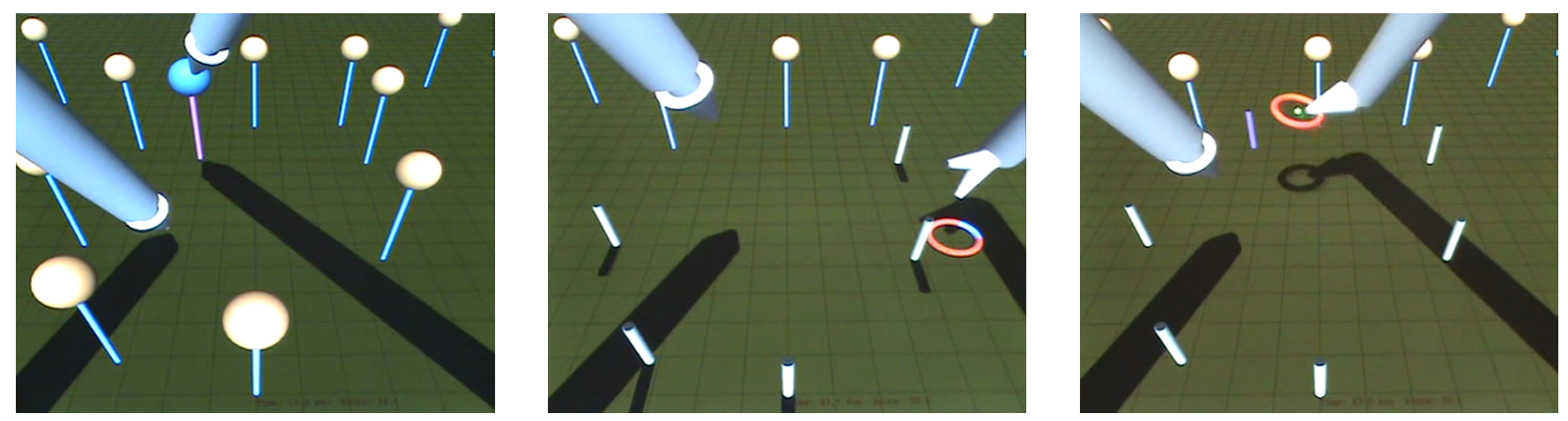

Fig. 2. Pointing and pick-and-place tasks in the virtual reality simulator: a) pointing task; b) grasping during pick-and-place task and c) approaching the destination pin during pick-and-place task 


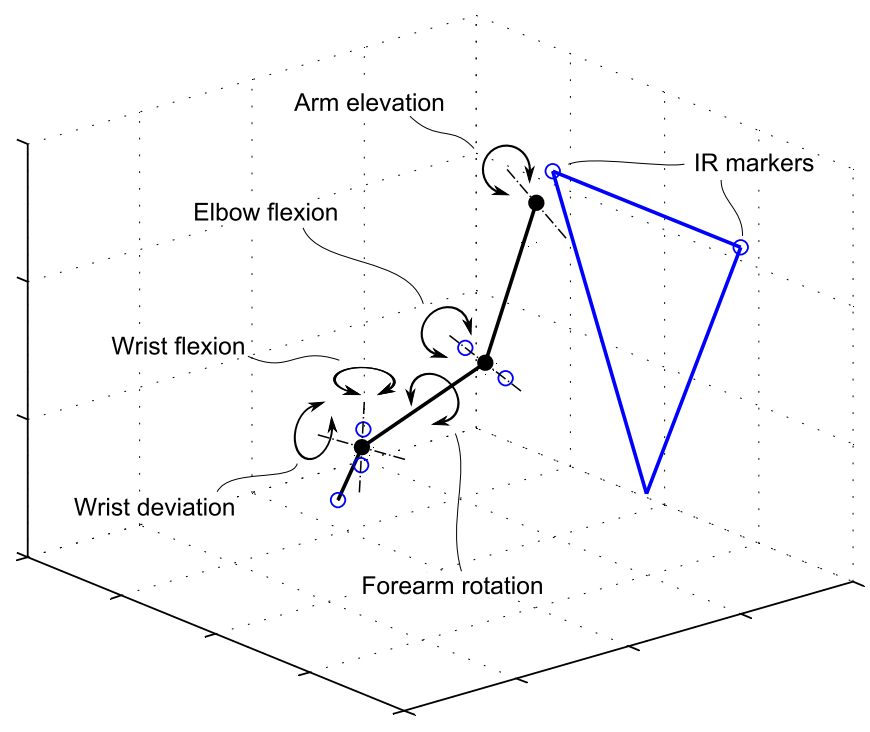

Fig. 3. Definition of upper limb angles used to compute the RULA-based ergonomic score; black-filled circles are the joint centers, empty circles are the markers positions

motions (in addition to global arm and forearm motions). Furthermore, the task is designed so that the use of intraabdominal mobilities is mandatory to succeed: the ring can only be grasped on one portion (in blue on Fig. 2b). As a consequence, it cannot be accomplished with a single straight instrument. This could be seen as a bias at the advantage of articulated instruments but we believe that it depicts the reality. Indeed, to prepare a suture when using standard instruments, a surgeon has to pass several times the needle from one instrument to the other to orient it properly. Therefore, a second straight instrument must be held by the subject to carry out the pick and place task.

As it is not usual for a surgeon to operate with one hand free, the subject had to hold this second straight instrument during all trials-both tasks with each instrument-, although it was unused most of the time.

\section{Metrics}

Gesture performance: Satava et al. [13] drew up a list of all laparoscopic skills assessment devices and metrics. It emerges from this review that gesture quality is usually quantified with a few simple metrics: time to completion, number of motions, number of errors, total distance traveled by the instrument tip, and spectrum of velocities and accelerations. A global index is also commonly used, made of a weighted sum of several metrics. We adopted a global performance index proposed by Huang et al. [14] that combines the time to completion $(T T C)$, the number of errors $(E r r)$ and the motion economy $(M E)$, defined as the ratio between actual and optimal (shortest) path length:

$$
\text { Performance }=T T C+E r r+2 \times M E .
$$

The weight for motion economy was chosen in agreement with surgeons, regarding the expected values for the two other metrics. An error is recorder each time an instrument
TABLE I

INDIVIDUAL POSTURE SCORES TO COMPUTE THE ERGONOMIC SCORE

\begin{tabular}{|c|c|}
\hline Upper-arm score & $\begin{array}{l}\text { Arm elevation angle } \\
\left(0^{\circ} \text { when arm down }\right)\end{array}$ \\
\hline 1 & $0-45^{\circ}$ \\
\hline 3 & $45-90^{\circ}$ \\
\hline 5 & $>90^{\circ}$ \\
\hline+1 & if shoulder is raised by $>10 \mathrm{~mm}$ \\
\hline Forearm score & $\begin{array}{l}\text { Elbow flexion angle } \\
\left(0^{\circ} \text { when arm and forearm aligned }\right)\end{array}$ \\
\hline 1 & $60-100^{\circ}$ \\
\hline 2 & $<60^{\circ} ;>100^{\circ}$ \\
\hline+1 & if hand crosses body midline or is out to side \\
\hline Wrist posture score & $\begin{array}{l}\text { Wrist flexion angle } \\
\left(0^{\circ} \text { when forearm and hand aligned }\right)\end{array}$ \\
\hline 1 & $-5-5^{\circ}($ neutral $)$ \\
\hline 2 & $-15--5^{\circ} ; 5-15^{\circ}$ \\
\hline 3 & $<-15^{\circ} ;>15^{\circ}$ \\
\hline+1 & if wrist deviation angle $>5^{\circ}$ \\
\hline Wrist twist score & Forearm rotation angle \\
\hline 1 & $-45-45^{\circ}$ \\
\hline 2 & $<-45^{\circ} ;>45^{\circ}$ \\
\hline
\end{tabular}

moves out of the screen (during both exercises) or when the ring collides with the target pin (pick and place task).

Ergonomic score: McAtamney and Corlett [15] proposed a method for quantifying the ergonomic level of work environment, called Rapid Upper Limb Assessment (RULA). The RULA score is calculated from the upper limb angles, general posture of trunk and neck, muscular load and time evolution of posture. The score ranges from 1 to 7 , increasing when ergonomic level decreases. Person et al. [16] established a real-time ergonomic index based on RULA, adapted to laparoscopic surgery. This index takes into account the movements of both arms, the trunk and the neck and is suitable for real-time motion tracking. We developed a similar RULA-based index that takes into account only the motions of the major arm (the one that holds the main instrument). Our ergonomics score comes from Table A of the original RULA score and ranges from 1 to 9 (the lower the better). It is obtained from 4 individual posture scores detailed in I: upper-arm score, forearm score, wrist posture score and wrist twist score. These individual posture scores are computed from joint angles defined in [17] and depicted in Fig. 3 . Joint angles are retrieved by means of a 3D localizer (see Section II-D). A set of 7 markers are placed on the subject (see Fig. 3): both shoulders (Acromioclavicular joint), major elbow (lateral and medial epicondyles), major wrist (radial and ulnar styloids), and major hand (metacarpal end of the middle finger). Center of rotation of wrist and elbow are supposed to be the midpoint of the segment joining the two corresponding markers. Center of the shoulder can be obtained using a spherefit algorithm with successive positions of one elbow marker (expressed in a frame attached on the subject's torso). Upper limb segments are then reconstructed between successive joint centers and angles values can be 


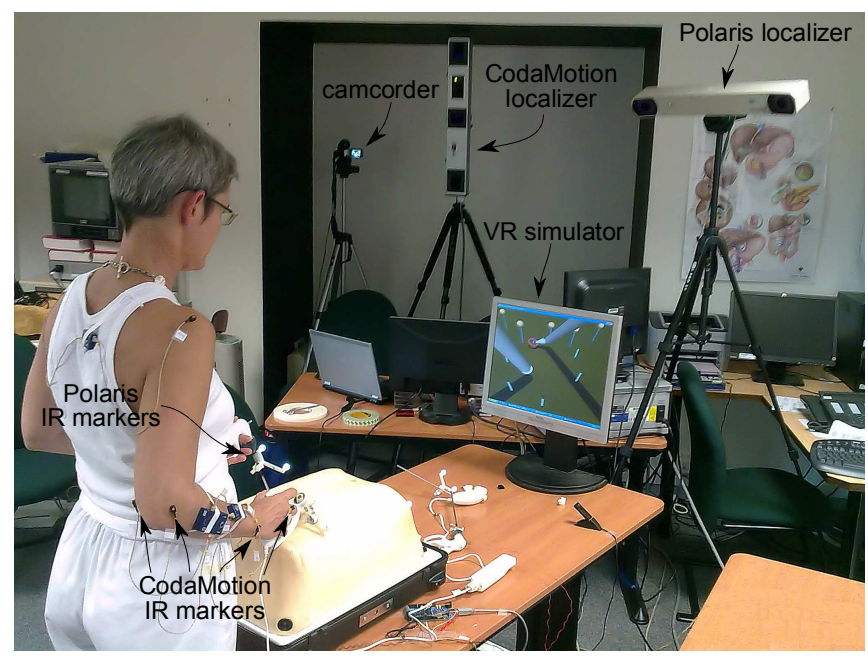

Fig. 4. Experimental setup during a trial

obtained by basic geometrical calculation.

\section{Experimental Setup}

The global experimental setup is shown in Fig. 4. Instruments mock-ups produced by rapid prototyping were inserted in a pelvitrainer through trocars that form a $10 \mathrm{~cm}$ equilateral triangle with a virtual laparoscope. Viewing angle of the latter was $45^{\circ}$ with respect to the table plane. A 19 " monitor was placed at $1.5 \mathrm{~m}$ from the subject's eyes, with a line of sight close to $25^{\circ}$ downwards. Table height was adjustable to fit with all subjects.

Exercises were implemented on a VR simulator. Instruments position and orientation were measured with a Polaris Optotrak system (Northern Digital Inc.) connected to the VR simulator via a serial port. This device can localize several targets with a precision of $0.3 \mathrm{~mm}$, using 2 infrared (IR) cameras, IR emitters and reflective spheres on each target.

Upper limb segments were tracked using a Codamotion system (Charnwood Dynamics Ltd.), made of a Hub computer unit and $3 \mathrm{Cx} 1$ units. A $\mathrm{Cx} 1$ unit comprises 3 IR sensors that track IR-emitting markers placed on the subject's arm. Each Cx1 unit is capable of retrieving the 3D position of all markers, but using 3 units improves accuracy $(<0.1 \mathrm{~mm})$ and provides redundancy. No interference occurred between Ploaris and Codamotion localizers.

All experiments were recorded using a camcorder placed in front of the subject.

\section{E. Protocol}

Two groups of subjects were recruited for the study:

- 10 surgeons from different specialties (urology, gynecology, general and digestive surgery) with various levels of expertise in laparoscopy and surgical robotics;

- $6 \mathrm{PhD}$ students in robotics with no experience in laparoscopy but used to playing video games.

During the placement and calibration of the Codamotion markers, the experiment purposes and methods were introduced to the subject. Emphasis was put on the fact that only instruments performance are measured and compared, with no intention to rank individuals. Subjects were told to perform each trial as quickly as possible, with the shortest path, and avoiding errors.

The experiment started with the standard laparoscopic instrument. A short demonstration was carried out to explain the pointing task. Then the subject repeated several times a learning exercise similar to the task before the recorded task itself, in order to get familiar with the VR environment, the task and the instrument. A learning curve was calculated after each learning exercise with the time and performance score and the subject could start the recorder trial only after reaching a learning plateau, to avoid learning during the trial. After the pointing trial, the same steps (demonstration, learning exercises, recorded task) were repeated for the pick and place task.

Each subject repeated this protocol for both dexterous instruments. These were used in alternate order among subjects in order to minimize the effect of learning the exercises with an instrument on the results with the other instrument.

\section{F. Statistical Analysis}

The performance score can be computed easily for each recorded trial. On the contrary, our RULA-based ergonomic score is instantaneous and computed for each Codamotion frame of a trial. The mean value of the ergonomic score (called 'RULA-mean' hereafter) was therefore calculated for each trial (as in [16]). In addition, standard deviation (RULA$\mathrm{SD}$ ) and minimum and maximum values (RULA-min and RULA-max) were also computed for each trial to have a more detailed view of the general ergonomic level.

For each task, an ANOVA was performed using Statistica to identify significant differences between instruments or subjects groups (students and surgeons), regarding either gesture or ergonomic performance. A complementary NewmanKeuls post-hoc test was performed when the ANOVA significance level was reached.

\section{RESULTS}

\section{A. Ergonomic Score}

Fig. 5a shows the mean values of the ergonomic scores (i.e. RULA-mean, RULA-SD, RULA-min, and RULA-max) computed for all trials of the pointing task, sorted by subjects group and instrument. Results for the pick and place task are summarized in Fig. $5 b$.

For the pointing task, the statistical analysis performed on RULA-mean does not show any significant difference between the 3 instruments. For surgeons however, the RULAmean score tends to be lower with the articulated handle, although it is not significant $(p>0.05)$. RULA-SD values show a significant effect of instrument $(F(2.26)=$ $7.1330, p<0.05)$, the articulated handle performing better than the other two), especially for students although there is no significant interaction between instrument and expertise. RULA-min values do not show any statistical trend. RULAmax values also show a significant effect of instrument $(F(2.26)=7.4533, p<0.05)$, with higher performance for 


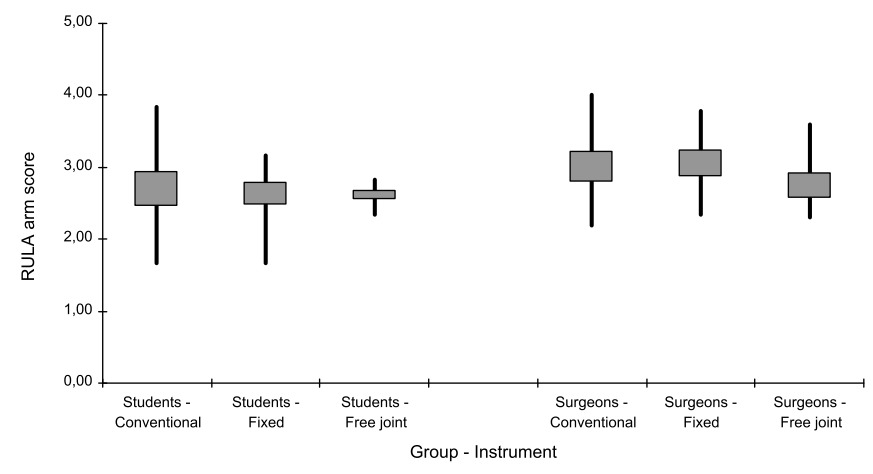

(a) Pointing task

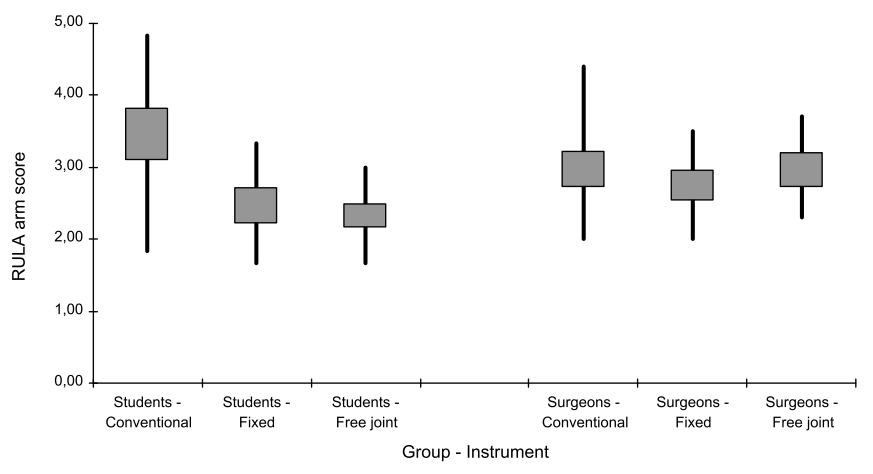

(b) Pick and place task

Fig. 5. RULA-based ergonomic scores; center of the box is the mean value of RULA-mean, height of the box is the mean value of RULA-SD, and limits of the segment are mean values of RULA-min and RULA-max

both robotized instruments, especially for students again but with no significant interaction.

For the pick and place task, RULA-mean values show a significant effect of instrument $(F(2.28)=17.1770, p<$ $0.05)$ and a significant interaction between instrument and expertise $(F(2.28)=11.8920, p<0.05)$. The same significant trend can be observed among both groups, standard instrument having lower performance that robotized instrument with fixed handle. However, a significant difference exists between surgeons and students regarding the articulated handle, which has the best performance for students but not for surgeons. RULA-SD values show a significant effect of instrument $(F(2.28)=3.8138, p<0.05)$ with articulated handle showing better performance. RULA-min values do not show any statistical trend. RULA-max values show a statistical advantage for articulated instruments for both groups $(p<0.05)$. There is a trend to interaction between instrument and group: the articulated handle has the best performance for students but not for surgeons.

\section{B. Gesture Performance}

Fig. $6 \mathrm{a}$ and $6 \mathrm{~b}$ show the mean value and standard deviation of the global performance score computed for all trials, sorted by subjects group and instrument, for the pointing and pick and place tasks respectively.

For the pointing task, the articulated handle is significantly less efficient $(F(2.26)=37.4650, p<0.05)$. There is a trend to interaction with expertise ( $p=0.06)$ : the fixed Nunchuk is the best for students, but do not perform significantly better than the standard instrument for surgeons.

For the pick and place task, there is a significant effect of instrument $(F(2.28)=4.9722, p<.005)$ with the fixed Nunchuck performing better that the other two.

\section{DISCUSSION}

From an ergonomic point of view, the novel solution of adding a free (passive) 3 DOF spherical joint between handle and shaft seems to be very effective. The instrument with the passive articulation is globally the most ergonomic one. All ergonomic criteria are clearly better with this instrument for the $\mathrm{PhD}$ student group. This is an interesting and encouraging result as none of the existing inner-DOF instruments has a solution to the problem of ergonomic shortage. Among surgeons, this trend is less visible and ergonomic scores are not as good as for roboticists. This can be explained by the fact that surgeons are used to working with the standard instruments: they have better ergonomic scores than novices for the complex pick and place task with such instruments.

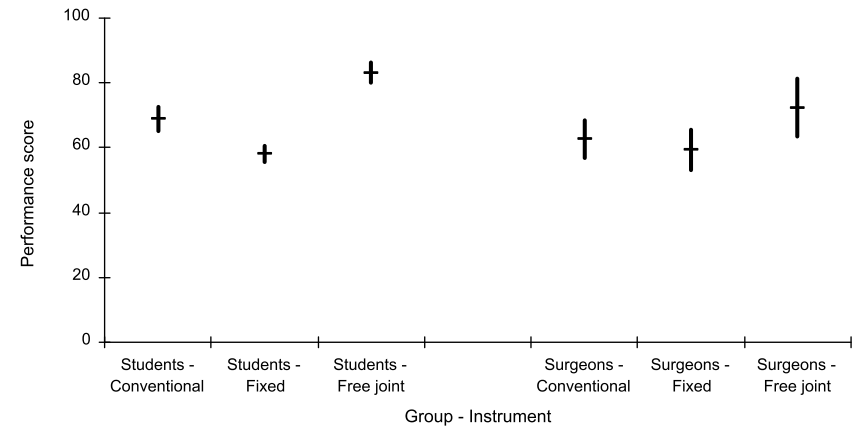

(a) Pointing task

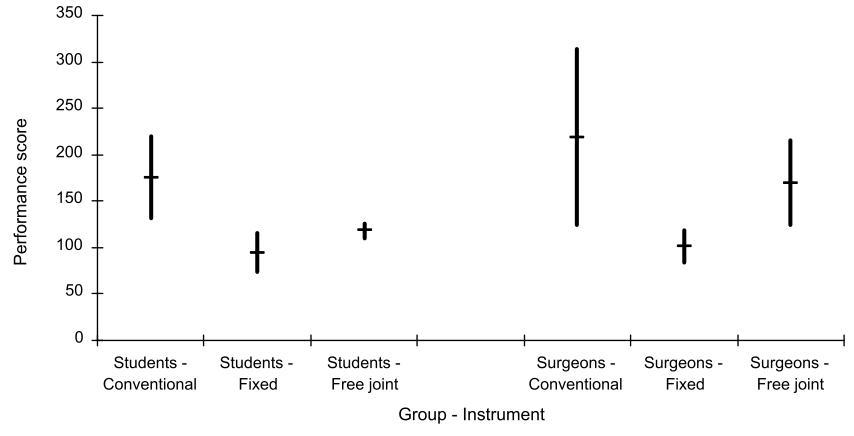

(b) Pick and place task

Fig. 6. Global performance scores; center of the segment is the mean value, and height of the segment is the standard deviation 
It is known in many fields (even outside medicine) that specialists experience some difficulties to replace a well known technique by a new one, as they have to unlearn their habits in order to exploit all capabilities offered by the new device. It can therefore be expected that, with an appropriate learning and training phase, surgeons will fill in the progression margin and perform as well as (or even better than) the students group.

From an efficiency point of view, the free joint handle is not the best solution among the 3 instruments. The dexterous instrument with a fixed handle is either the most efficient or as efficient as the conventional one. This is because controlling accurately the position of the end-effector is more difficult with the free joint. This clearly influences the results for the pointing task and could better be visualized through a spectral analysis of speeds and accelerations. Another disadvantage of the free handle is that it requires one more actuator-and consequently, one more control means - than with a fixed one, as explained in Section II-A. This increases the complexity of control, as we could notice during the experimental campaign. Subjects that could easily drive the 2 intra-abdominal DOF with the fixed Nunchuk became hesitant with this additional DOF. This adds up with the precision problem and explains the rather poor results during the pick and place task. However, one can notice that both groups performed better with distal mobilities during the manipulation task. This demonstrates the benefits of intraabdominal mobilities for complex surgical gestures. One surprising point is that students performed globally better than surgeons for the pick and place task, regardless the instrument. This can probably be explained by the fact that our VR simulator is rather removed form surgical reality: absence of anatomical landmarks, no perception of interaction forces between tool and tissues. This can unsettle expert surgeons a bit while $\mathrm{PhD}$ students in robotics consider the VR interface as a video game that they are used to learn quickly.

\section{COnClusions And Future Work}

To solve a part of the lack of ergonomics in laparoscopic surgery and facilitate complex gestures, a novel instrument was designed. It comprises an articulated end-effector with Yaw-Roll intra-abdominal mobilities, and an ergonomic handle that is linked to the shaft through a passive spherical joint. Both ergonomic and gesture performance of this new instrument were assessed through a series of experiments on a VR simulator. 10 laparoscopists and $6 \mathrm{PhD}$ students in robotics took part in the study.

It is shown that the additional mobilities improve performance (i.e. completion time, economy of movement) for complex tasks. The articulated handle allows for working in a more ergonomic way but requires a learning and adaptation phase to reach the same level of performance. It could be of interest to study more deeply the role of learning exercises on surgical performance with a novel instrument that modifies noticeably experts' habits. For example, the evaluation trials could be repeated several times during a 2 weeks learning and training protocol with the new instruments, and several weeks after the training phase.

It would also be worth trying to combine the advantages of the articulated handle in terms of ergonomic quality with the performance of the same instrument with a fixed handle. A solution might be to provide a handle that could be adjusted freely during large instrument repositioning and locked afterwards for performing fine gestures in a narrow workspace, with little changes in the arm posture.

Finally, the experimental setup should also be updated to offer force feedback. This seems more important than designing an anatomical environment, as most surgeons are used to train on pelvitrainer.

\section{ACKNOWLEDGEMENT}

The authors would like to thank warmly all surgeons at Institut Mutualiste Montsouris and students at Institut des Systèmes Intelligents et de Robotique who took part in the experiments.

\section{REFERENCES}

[1] A. Cuschieri, "Whither minimal access surgery: tribulations and expectations," The American Journal of Surgery, vol. 169, no. 1, pp. 9-19, jan 1995.

[2] R. Berguer, D. L. Forkey, and W. D. Smith, "Ergonomic problems associated with laparoscopic surgery," Surgical Endoscopy, vol. 13, no. 5, pp. 466-468, May 1999.

[3] A. Vereczkei, H. Feussner, T. Negele, F. Fritzsche, T. Seitz, H. Bubb, and O. P. Horváth, "Ergonomic assessment of the static stress confronted by surgeons during laparoscopic cholecystectomy," Surgical Endoscopy, vol. 18, no. 7, pp. 1118-1122, July 2004.

[4] R. Berguer, "Surgical technology and the ergonomics of laparoscopic instruments," Surgical Endoscopy, vol. 12, no. 5, pp. 458-462, May 1998.

[5] T. Emam, T. Frank, G. Hanna, and A. Cuschieri, "Influence of handle design on the surgeon's upper limb movements, muscle recruitment, and fatigue during endoscopic suturing," Surgical Endoscopy, vol. 15, no. 7, pp. 667-672, July 2001.

[6] N. T. Nguyen, H. S. Ho, W. D. Smith, C. Philipps, C. Lewis, R. M. D. Vera, and R. Berguer, "An ergonomic evaluation of surgeons' axial skeletal and upper extremity movements during laparoscopic and open surgery," The American Journal of Surgery, vol. 182, no. 6, pp. 720724, Dec. 2001.

[7] U. Matern and P. Waller, "Instruments for minimally invasive surgery: principles of ergonomic handles," Surgical Endoscopy, vol. 13, no. 2, pp. 174-182, Feb. 1999.

[8] A. H. Zahraee, B. Herman, and J. Szewczyk, "Mechatronic design of a hand-held instrument with active trocar for laparoscopy," in Proc. IEEE International Conference on Robotics and Automation (ICRA'11), 2011, accepted for publication.

[9] A. H. Zahraee, J. K. Paik, J. Szewczyk, and G. Morel, "Toward the development of a hand-held surgical robot for laparoscopy," IEEE/ASME Trans. Mechatron., vol. 15, no. 6, pp. 853-861, dec 2010.

[10] S. Ahmed, G. Hanna, and A. Cuschieri, "Optimal angle between instrument shaft and handle for laparoscopic bowel suturing," Archives of Surgery, vol. 139, no. 1, pp. 89-92, Jan. 2004.

[11] A. M. Derossis, G. M. Fried, M. Abrahamowicz, H. H. Sigman, J. S. Barkun, and J. L. Meakins, "Development of a model for training and evaluation of laparoscopic skills," The American Journal of Surgery, vol. 175, no. 6, pp. 482-487, June 1998.

[12] C. G. L. Cao, C. L. Mackenzie, and S. Payandeh, "Task and motion analyses in endoscopic surgery," in Proc. ASME/IMECE Annual Symposium on Haptic Interfaces for Virtual Environment and Teleoperator Systems, 1996, pp. 583-590.

[13] R. Satava, A. Cuschieri, and J. Hamdorf, "Metrics for objective assessment," Surgical Endoscopy, vol. 17, no. 2, pp. 220-226, Feb. 2003. 
[14] J. Huang, S. Payandeh, P. Doris, and I. Hajshirmohammadi, "Fuzzy classification: towards evaluating performance on a surgical simulator," in Proc. Medicine Meets Virtual Reality (MMVR'05), 2005, pp. 194200.

[15] L. McAtamney and E. N. Corlett, "RULA: a survey method for the investigation of work-related upper limb disorders," Applied Ergonomics, vol. 24, no. 2, pp. 91-99, Apr. 1993.

[16] J. Person, A. Hodgson, and A. Nagy, "Automated high-frequency posture sampling for ergonomic assessment of laparoscopic surgery," Surgical Endoscopy, vol. 15, no. 9, pp. 997-1003, 2001.

[17] D. Romilly, C. Anglin, R. Gosine, C. Hershler, and S. Raschke, "A functional task analysis and motion simulation for the development of a powered upper-limb orthosis," IEEE Trans. Rehab. Eng., vol. 2, no. 3, pp. 119-129, Sept. 1994. 